\title{
Local analogs of high-redshift galaxies: Metallicity calibrations at high-redshift
}

\author{
Fuyan Bian ${ }^{1}$, , Lisa J. Kewley ${ }^{2}$, Brent Groves ${ }^{2}$ and \\ Michael A. Dopita ${ }^{2}$ \\ ${ }^{1}$ European Southern Observatory, Alonso de Córdova 3107, Casilla 19001, \\ Vitacura, Santiago 19, Chile \\ email: fbian@eso.org \\ ${ }^{2}$ Research School of Astronomy and Astrophysics, Australian National University, Canberra, \\ ACT 2611, Australia
}

\begin{abstract}
We study the metallicity calibrations in high-redshift galaxies using a sample of local analogs of high-redshift galaxies selected from the SDSS survey. Located in the same region on the BPT diagram as star-forming galaxies at $z \sim 2$, these high-redshift analogs share the same ionized ISM conditions as high-redshift galaxies. We establish empirical metallicity calibrations between the direct gas-phase oxygen abundances and varieties of metallicity indicators in our local analogs using direct $T_{e}$ method. These new metallicity calibrations are the best means to measure the metallicity in high-redshift galaxies. There exist significant offsets between these new high-redshift metallicity calibrations and local calibrations. Such offsets are mainly driven by the evolution of the ionized ISM conditions from high-z to low-z.
\end{abstract}

Keywords. galaxies: high-redshift, ISM: abundances, galaxies: abundances, galaxies: ISM

\section{Introduction}

The gas-phase oxygen abundance is not only modulated by the metal enrichment from massive stars, but also by the physical processes of outflow and inflow in galaxies. Studying gas-phase oxygen abundance provides great insight into the key physical processes that control the formation and evolution of galaxies. By studying the evolution of gas-phase chemical abundance depending on stellar mass (mass-metallicity relation), people can put strong constraints on galaxy build-up process and the stellar feedback models that drive galactic-scale outflows (e.g. Lilly et al. 2013). Therefore, it is essential to measure the chemical abundance accurately to study the metallicity evolution in galaxies over cosmic time.

Metallicity indicators based on metallicity sensitive nebular emission-line ratios are widely used to estimate the gas-phase oxygen abundance in galaxies and H II regions. These metallicity indicators are calibrated based on either photoionization models (e.g., Kewley \& Dopita 2002) or empirical calibrations (Pettini \& Pagel 2004). In particular, metallicity measurements in high-redshift galaxies heavily rely on the empirical calibrations, which are usually calibrated to $\mathrm{H}$ II regions in nearby star-forming galaxies using the metallicity derived using direct $T_{e}$ method.

Photoionization models suggested the ionized ISM conditions, including the ionization parameter, the electron density, the nitrogen-to-oxygen ratio $(\mathrm{N} / \mathrm{O})$, and the spectral shape of the radiation field, can affect the metallicity sensitive strong emission-line ratios (e.g., Kewley et al. 2013). Observational evidence suggests that the ISM conditions change dramatically over cosmic time Steidel et al. 2014. For example, the ISM conditions in high-redshift star-forming galaxies have $\sim 0.6$ dex higher ionization parameters and an 

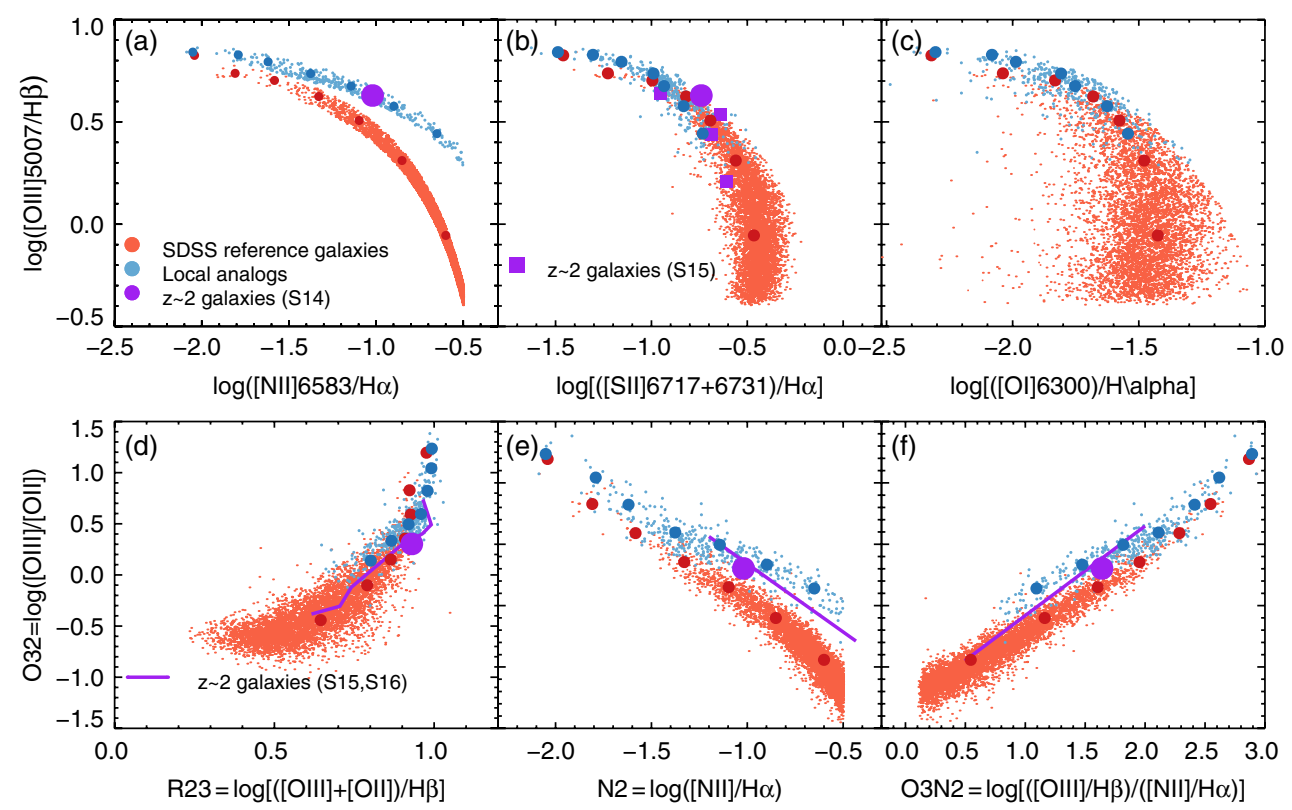

Figure 1. Optical diagnostic diagrams of local analogs of high-redshift galaxies and SDSS reference galaxies. The small blue and red points represent the individual local analogs and SDSS reference galaxies, respectively, and the large open blue and red points represent the stacked spectra of the local analogs and SDSS reference galaxies, respectively. The large purple filled circle represents the stacked spectrum of a sample of $z \sim 2 \mathrm{UV}$-selected galaxies adopted from Steidel et al. 2014. The figure is adopted from Bian et al. 2018.

order of magnitude higher electron densities than their local counterparts (Bian et al. 2010).

The strong evolution of the ionized ISM raises the following question: Are the local metallicity calibrations still applicable for high redshift galaxies? We approach this issue by empirically calibrating the metallicity indicators in a sample of local analogs of highredshift galaxies using the direct $T_{e}$ method. We compare the empirical calibrations derived from the local analogs with those derived from a sample of local reference galaxies to study how metallicity calibrations change with cosmic time.

\section{Sample Selection}

We select local analogs of high-redshift galaxies from the SDSS MPA-JHU catalog based on their locations on the BPT diagram. These local analogs are selected in the \pm 0.04 dex region of the $z \sim 2.3$ star-forming sequence defined by equation 9 in Steidel et al. 2014 on the BPT diagram (small blue data points in Figure 1(a)). Our studies have shown that this type of local analogs closely resembles the properties of the highredshift galaxies (Bian et al. 2016, Bian et al. 2017). In particular, these analogs have high ionization parameters $\left(\log q \simeq 7.9 \mathrm{~cm}^{-1}\right)$ and high electron densities $\left(n_{e} \simeq 120 \mathrm{~cm}^{-3}\right)$, which are an order of magnitude higher than local star-forming galaxies. These properties are comparable to those in the $z \sim 2-3$ galaxies (e.g., Nakajima et al., Sanders et al. 2016). These local analogs also share the same region with $z \sim 2$ star-forming galaxies in all the optical diagnostic diagrams (Figure 1).

To compare the metallicity calibrations between the local analogs and nearby galaxies, we also select local reference galaxies located in the \pm 0.05 dex region of the local starforming sequence on the BPT diagram. 


\section{Direct $T_{e}$ Metallicity}

To detect the [O III $] \lambda 4363$ line, we generate composite spectra for both the local analogs and SDSS reference galaxies by stacking the individual spectra in different $[\mathrm{N} \mathrm{II}] \lambda 6584 / \mathrm{H} \alpha$ bins. First of all, we use the Balmer decrement to correct the dust extinction in the reduced 1D galaxy spectra from the SDSS DR9. Then we shift each of the spectra to the rest-frame wavelength based on their redshifts and resample the spectra onto a grid of wavelength from $3700 \AA$ to $7300 \AA$ with $\Delta \lambda=1 \AA$. The mean flux density in the wavelength range of $4400-4450 \AA$ is used to normalize the spectra. The local analogs and the SDSS redshift galaxies are divided into 0.25 dex bins in $[\mathrm{N}$ II] $] \lambda 6584 / \mathrm{H} \alpha$ from $\log ([\mathrm{N} \mathrm{II}] \lambda 6584 / \mathrm{H} \alpha)=x$ to $x+0.25$, where $x=[-2.25,-2.00,-1.75,-1.50,-1.25$, $-1.00,-0.75]$. At last, we stack the spectra within each of the $[\mathrm{N} \mathrm{II}] \lambda 6584 / \mathrm{H} \alpha$ bins using the mean flux density at each wavelength, and a total of fourteen stacked spectra are generated: seven spectra of local analogs of high-redshift galaxies and seven spectra of SDSS reference sample.

We subtract the stellar continuum from the composite spectra using the STARLIGHT stellar population synthesis models. Then the fluxes of the $[\mathrm{O}$ III $] \lambda 4363$ lines are measured by fitting with a Gaussian function. To get reliable direct $T_{e}$ oxygen abundance measurements, we only adopt the stacked spectra with signal-to-noise ratios $(\mathrm{S} / \mathrm{N})$ of $[\mathrm{O}$ III $] \lambda 4363$ greater than ten $(\mathrm{S} / \mathrm{N}>10)$. After this $\mathrm{S} / \mathrm{N}$ cut, six composite spectra for local analogs and four composite spectra for SDSS reference galaxies are available for our further analysis.

We use the Izotov et al. 2006 recipe to estimate the electron temperature in the $\mathrm{O}^{++}$zone $\left(T_{e}(\mathrm{O}\right.$ III $\left.)\right)$ using $[\mathrm{O}$ III $] \lambda \lambda 4959,5007 /[\mathrm{O}$ III $] \lambda 4363$ ratio. The following relation between $T_{e}(\mathrm{O}$ III $)$ and $T_{e}(\mathrm{O}$ II $)$ is adopted from Garnett 1992: $T_{e}(\mathrm{O}$ II $)=0.7 T_{e}(\mathrm{O}$ III $)$ $+3000 \mathrm{~K}$. The $\mathrm{O}^{++}$abundance is estimated using the $T_{e}(\mathrm{O}$ III $)$ temperature and $[\mathrm{O}$ III $] \lambda \lambda 4959,5007 / \mathrm{H} \beta$ ratio, and $\mathrm{O}^{+}$abundance is estimated using the $T_{e}(\mathrm{O}$ II $)$ temperature together with $[\mathrm{O} \mathrm{II}] \lambda \lambda 3726,3729 / \mathrm{H} \beta$ and electron density. The final oxygen abundance is the sum of the $\mathrm{O}^{++}$abundance and the $\mathrm{O}^{+}$abundance.

\section{Results}

We use the direct $T_{e}$ metallicity and line ratios measured in the stacked spectra to calibrate varieties of metallicity indicators, including N2, O3N2, R23, O32, $\log$ ([OIII] 5007 / $\mathrm{H} \beta)$, and $\log ([\mathrm{NeIII}] 3869 /[\mathrm{OII}] 3727)$. Figure 2 shows the relations between the direct $T_{e}$ oxygen abundance and the above metallicity indicators in the local analogs of highredshift galaxies (blue squares) and the local normal star-forming galaxies (red squares). There are significant offsets between the local analogs and SDSS reference galaxies.

We use a linear equation to fit the relation between the direct oxygen abundance and the N2, O3N2,O32, and $\log ([\mathrm{NeIII}] 3869 /[\mathrm{OII}] 3727)$ indicators in local analogs. The results are as follows:

$$
\begin{array}{r}
12+\log (\mathrm{O} / \mathrm{H})=8.82+0.49 \times \mathrm{N} 2, \\
12+\log (\mathrm{O} / \mathrm{H})=8.97-0.39 \times \mathrm{O} 3 \mathrm{~N} 2, \\
12+\log (\mathrm{O} / \mathrm{H})=8.54-0.59 \times \mathrm{O} 32 . \\
12+\log (\mathrm{O} / \mathrm{H})=7.80-0.63 \times \log ([\mathrm{NeIII}] /[\mathrm{OII}]) .
\end{array}
$$

For the R23-Z relation, our data only cover the R23 upper branch. Therefore, a thirdorder polynomial is used to fit the upper branch of the $\mathrm{R} 23-\mathrm{Z}$ relation:

$$
y=138.0430-54.8284 x+7.2954 x^{2}-0.32293 x^{3}
$$

where $y=R 23$ and $x=12+\log (\mathrm{O} / \mathrm{H})$. 

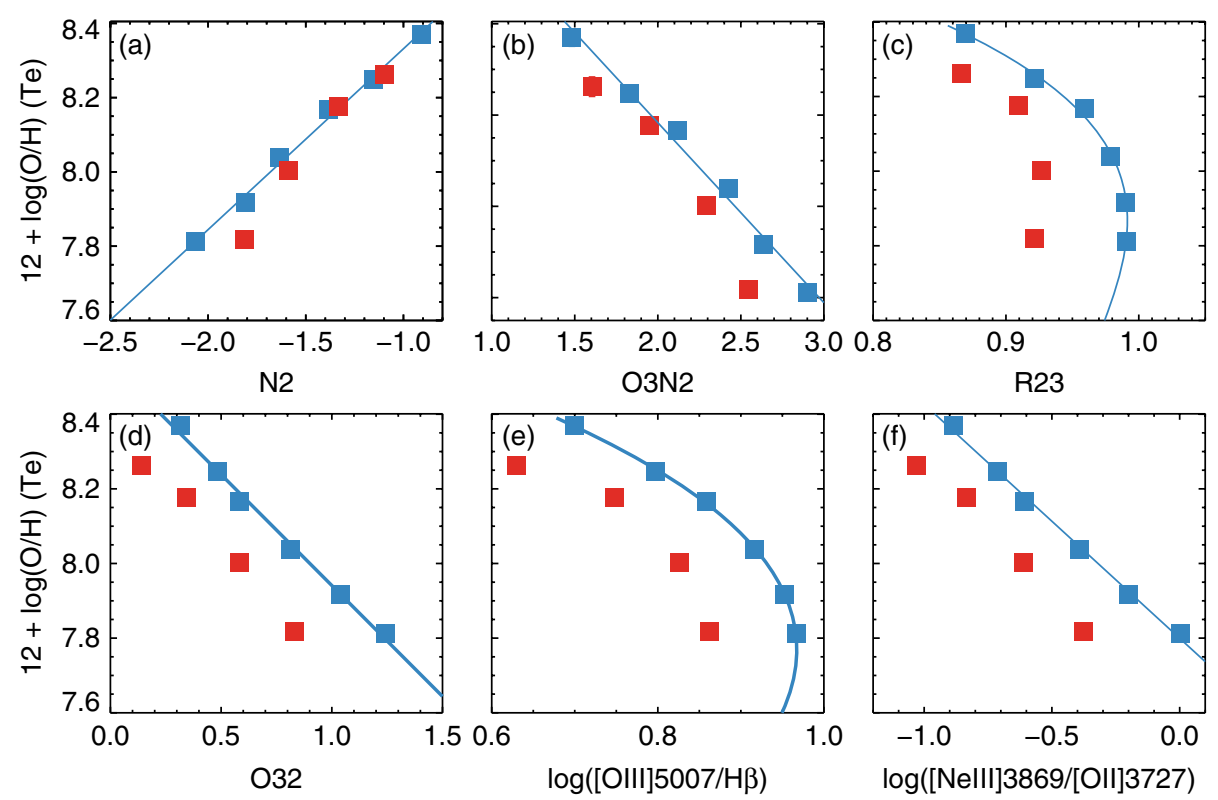

Figure 2. Metallicity calibrations of different metallicity indicators using direct $T_{e}$ method in the local analogs of high-redshift galaxies (blue data points) and the SDSS reference galaxies (red data points).

For the $[\mathrm{O}$ III $] \lambda 5007 / \mathrm{H} \beta$-Z relation, we also use a third-order polynomial to fit the data points:

$$
y=43.9836-21.6211 x+3.4277 x^{2}-0.1747 x^{3}
$$

where $y=[\mathrm{O}$ III $] \lambda 5007 / \mathrm{H} \beta$ and $x=12+\log (\mathrm{O} / \mathrm{H})$.

It is worth noting that our new metallicity calibrations for high redshift galaxies are ONLY valid in the metallicity range of 7.8 to 8.4. The blue solid curves in Figure 2 show the best-fitted results for each of the metallicity indicator. Using a sample of $z \sim 2-3$ galaxies with direct oxygen abundance, Sanders et al. 2019 suggested that this new set of the metallicity calibrations is the best approach to measure the metallicity in highredshift galaxies. Especially in the JWST era, these calibrations can be used to measure metallicity in star-forming galaxies at $z>6$.

\section{Discussion}

The relations between the oxygen abundance and metallicity indicators for two cases are established based on the MAPPINGs photoionization models: 1 . The first model with the ionization parameter of $\log q=7.5$ and the ISM pressure is $\log (P / k)=5.2$. This model represents the ionized conditions in nearby galaxies (Bian et al. 2016). 2 . The second model with the ionization parameter of $\log q=8.0$ and the ISM pressure of $\log (P / k)=6.2$. These ISM conditions are consistent with those in $z \sim 2$ galaxies and our local analogs (Kaasinen et al. 2017). The relations between metallicity sensitive diagnostic line ratios and the oxygen abundance derived from the photoionization models are shown in Figure 3. Though there exist large offsets on the absolute value of the metallicity estimation between the metallicities derived from empirical calibrations and photoionization models, the metallicity calibrations between the high-redshift and lowredshift conditions derived by the photoionization models (solid lines in Figure 3) follow the same trend as those between the local analogs and the SDSS reference galaxies 

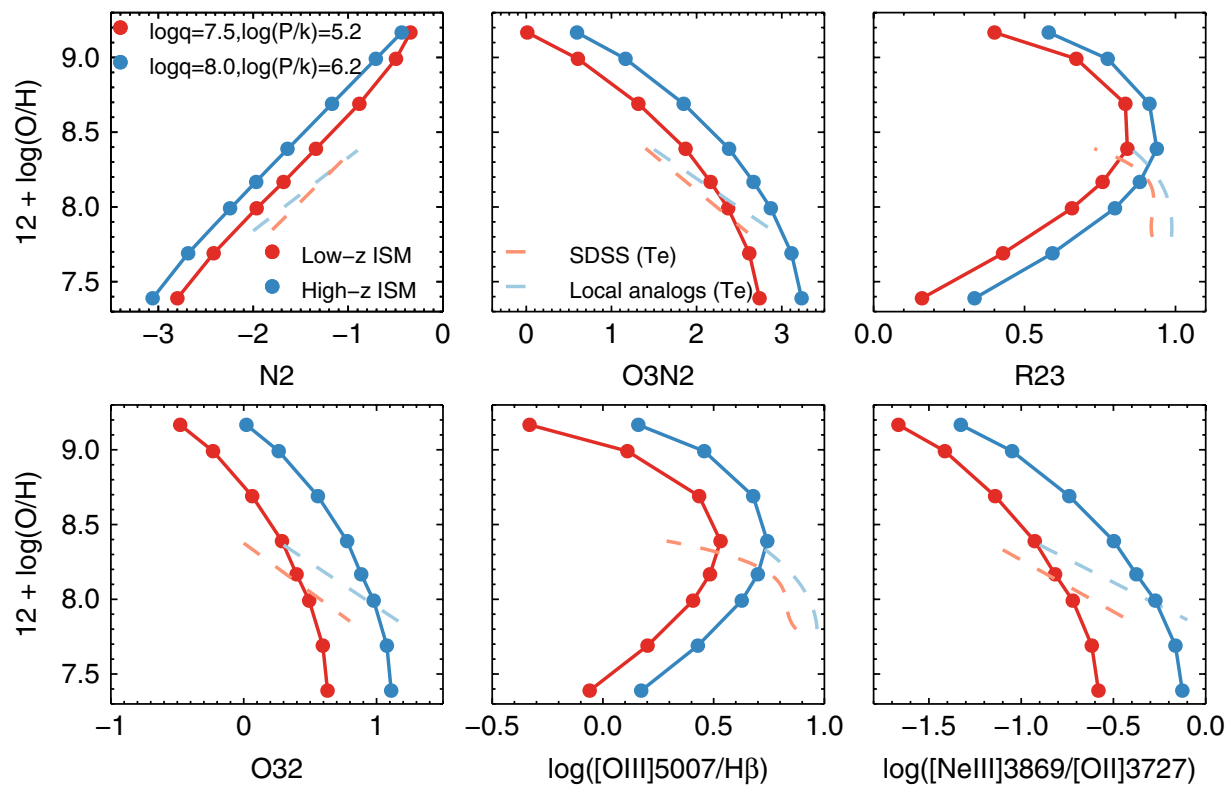

Figure 3. Relations between metallicity indicators and oxygen abundance from the MAPPINGs photoionization models. The red data points represent the photoionization models with ISM pressure $\log (P / k)=5.2$ (electron density of $n_{e} \sim 10$ ) and photoionization parameter of $\log q=$ 7.5 , which are representative values found in local star-forming galaxies. The blue data points represent the photoionization models with ISM pressure $\log (P / k)=6.2$ (electron density of $n_{e} \sim$ 100 ) and photoionization parameter of $\log q=8.0$, which are comparable to those in $z \sim 2$ starforming galaxies and our local analogs. The dashed lines represent the metallicity calibrations derived from the direct $T_{e}$ method in the SDSS reference galaxies (light red dashed line) and the local analogs of high-redshift galaxies (light blue dashed line). The figure is adopted from Bian et al. 2018.

(dashed lines in Figure 3). This suggests that the change of the metallicity calibration relations between the high- and low-redshift galaxies are due to the evolution of the ISM conditions.

\section{References}

Bian, F., Fan, X., Bechtold, J., et al. 2010, ApJ, 725, 1877

Bian, F., Kewley, L. J., Dopita, M. A., et al. 2018, ApJ, 859, 175

Bian, F., Kewley, L. J., Dopita, M. A., et al. 2017, ApJ, 834, 51

Bian, F., Kewley, L. J., Dopita, M. A., et al. 2016, ApJ, 822, 62

Garnett, D. R. 1992, AJ, 103, 1330

Izotov, Y. I., Stasińska, G., Meynet, G., et al. 2006, A $\& A, 448,955$

Kaasinen, M., Bian, F., Groves, B., et al. 2017, MNRAS, 465, 3220

Kewley, L. J. \& Dopita, M. A. 2002, ApJS, 142, 35

Kewley, L. J., Maier, C., Yabe, K., et al. 2013, ApJ, 774, L10

Lilly, S. J., Carollo, C. M., Pipino, A., et al. 2013, ApJ, 772, 119

Nakajima, K. \& Ouchi M. 2014, MNRAS, 442, 900

Pettini, M. \& Pagel, B. E. J. 2004, MNRAS, 348, L59

Sanders, R. L., Shapley, A. E., Kriek M., et al. 2016, ApJ, 816, 23

Steidel, C. C., Rudie, G. C., Strom, A. L. et al. 2014, ApJ, 795, 165 